\title{
Challenges to identify and mentor gifted children in developing countries: the Indian experience
}

\begin{abstract}
Anitha Kurup
The National Education Policy 2020 (NEP-2020) has recognized the need to identify and nurture gifted and talented children beyond the school curriculum. Through systematic research, the National Institute of Advanced Studies, Bengaluru has developed multiple protocols for identifying and multi-level, multi-stage mentoring of gifted children. This study argues that there is need for a paradigm shift in the identification process that predominantly uses psychometric measurements in the portfolio that captures the actual data points of gifted children. This is particularly relevant for developing countries like India, which has a diverse population predominantly from disadvantaged communities. Establishing norms for standardized psychometric tests developed for the western populations will have inherent limitations to identifying the gifted children in multi-lingual and multi-cultural contexts. Needless to state, India has far too few trained psychologists to accomplish this task.
\end{abstract}

Keywords: Developing countries, gifted children, identification, mentoring, psychometric measurements.

'GIFTED individuals are those who demonstrate outstanding levels of aptitude (seen as exceptional ability to reason and learn) or competence (documented performance or achievement in top $3 \%$ or rarer) in one or more domains. Domains include any structured area of activity with its own symbol system (e.g. mathematics, music, language) and/or set of sensory motor skills (e.g. painting, dance and sports). They must demonstrate the intentions of using the above abilities for constructive personal and/or social purpose ${ }^{1}$.'

Students who are gifted and talented often remain neglected in India. Since these children have exceptional abilities, they demonstrate special needs during the period of their development. Gifted children have high cognitive and emotional needs which are not met in usual classrooms geared to the average learner ${ }^{2,3}$. The fact that these children are in the minority adds to the problem of alienation and a sense of loss of direction. Both teachers and parents of these children struggle to fulfil their learning requirements in terms of information, resources, providing opportunities and extending emotional support to them.

The National Education Policy (NEP) 2020 has for the first time brought to the centre-stage the need to identify innate talents of students, which must be discovered, nur-

Anitha Kurup is in the National Institute of Advanced Studies, Bengaluru 560 012, India.

e-mail: bkanitha@nias.res.in tured, fostered, and developed beyond the school curricu$\operatorname{lum}^{4}$. Teacher education will include methods for recognition and fostering of talents and interests of these children including specialization in the education of the gifted in the newly proposed four-year integrated pre-service training to be implemented before 2030 . Casting a wide net for providing mentoring to the gifted children, the document enlists project-based clubs and circles at the school and district levels. Recognizing the challenge of distance in rural and tribal areas, provision for transport through funding will be provided to include socio-economically disadvantaged children in the programme. Leveraging technology to provide access to enriched curriculum and to mentoring opportunities is envisaged to reach out to the gifted children from disadvantaged communities ${ }^{4}$.

For the last few years, the National Institute of Advanced Studies (NIAS), Bengaluru has been working on issues of critical importance in education. The nature/nurture debate around giftedness continues with little conclusive evidence on the mutual influences of innate ability and environmental impacts on gifted individuals. Research on giftedness over the last several decades spans multiple geographies and diverse populations in extremely different contexts that result in differential opportunities. The variations in opportunities available to gifted children have a direct bearing on the manifestations of behaviour associated with giftedness as well as nurturance of the gift. Thus, research at NIAS has 
brought to the centre stage the behaviour displayed by gifted children and the contexts that provided opportunities for them to display that particular behaviour.

The NIAS student nomination behaviour rating scale was developed through $568 \mathrm{~h}$ of classroom observations across different school settings ${ }^{5}$. Such intensive observations were necessary to capture the complex interconnections between gifted behaviour and the contexts that offer the opportunities to demonstrate such behaviour. Informed by global repositories of the literature and research, we sought to move away from frameworks developed in the West to identify gifted children, where the identification rests largely on psychometric measurements. India, like many other developing countries, has to deal with millions of children who are gifted and hence there was a need to develop a tool based on observed behaviour in relation to its context. While the validity and reliability of the tool have to be established against different populations, it is also important to note that data on observed behaviour need to be generated on a large scale in order to study the emerging patterns across different geographies. Teachers and parents are thus central to this nomination process because they have predominant access to the children.

Building on the student nomination behavioural rating scale, it is imperative to develop a case study and a comprehensive portfolio of children from disadvantaged communities, to enable a fair comparison of their abilities with children from similar backgrounds. In developing countries, populations have different circumstances, both with respect to resources as well as school environments. This questions the usability of standardized identification methods for a heterogenous population for which they were not developed. Variations in schools are found in curriculum, teacher quality, teaching learning material and classroom processes. School variations are accompanied by varying home backgrounds which manifest in differential social capital and also have a bearing on gifted behaviour. Opportunities for identification of the gifted are highly correlated to behaviour shown in relation to the specific contexts. It is this symbiotic relationship between behavioural characteristics and specific contexts that compels one to recognize that standardized tools may have serious limitations in extremely different environments. In other words, it may be important to argue that gifted children from disadvantaged communities suffer similar challenges as gifted children who are differently abled. Hence, the portfolio approach with comprehensive real data points is a useful starting point. Large-scale data generation from different communities allows us to analyse patterns that may lend themselves to standardization processes. This is of great significance to gifted children in developing countries, since a predominant number of them may belong to disadvantaged communities. This is also crucial to address the low representation of marginalized communities in gifted education programmes. Additionally, there is a need to recognize that most developing countries have inadequate resources - both human and financial - to embrace the identification methods adopted by the developed countries. The small number of trained psychologists and insufficient resources to support standard psychometric measurements will drive low- and middle-income countries to adopt identification procedures that are commensurate with their resources.

Keeping the above factors in mind, the NIAS Programme on Education for the Gifted and Talented (NIAS-EGT) has developed multiple protocols to identify gifted children in both rural and urban populations. Figure 1 highlights the multiple identification protocols developed over the last decade.

\section{Identification protocols}

NIAS-EGT has developed three different protocols for the identification of gifted and talented children. (1) NIAS General Pool (0-15 years). (2) NIAS-supported Advance Learning Centres (11-15 years). (b) NIASPRODIGY-MAIYA Fellowship Programme (NMMFP; 16-18 years). (3) (a) NIAS-supported Advanced Learning Centres for the Urban Poor (11-15 years). (b) NIAS Mentoring Programme for Rural and Adivasi communities (11-15 years).

\section{NIAS general pool (0-15 years)}

NIAS has launched a May I Help You Centre through the website http://www.prodigy.net.in, where teachers or parents can nominate a child for the NIAS Gifted Education Programme. The nomination is made through a teacher/ parent nomination scale that needs to be downloaded, filled and e-mailed to the organization. Based on the information provided in this form, the team will solicit additional data points with regard to the student. These data help develop a case study and portfolio on the child. This identification protocol is available for children in the age group 3-15 years. NIAS is currently conducting research to identify children in the age group 0-3 years. Research has pointed out the need for identification of gifted children and appropriate intervention during the early years ${ }^{6-8}$.

The detailed case profile will contain:

- Individual factors (intellectual, cognitive and personality factors).

- Socio-cultural factors that influence the development of giftedness.

Interactions with parents, extended families, community members/networks and teachers are carried out. This is because the community and peers of gifted children 


\section{Identification protocols}
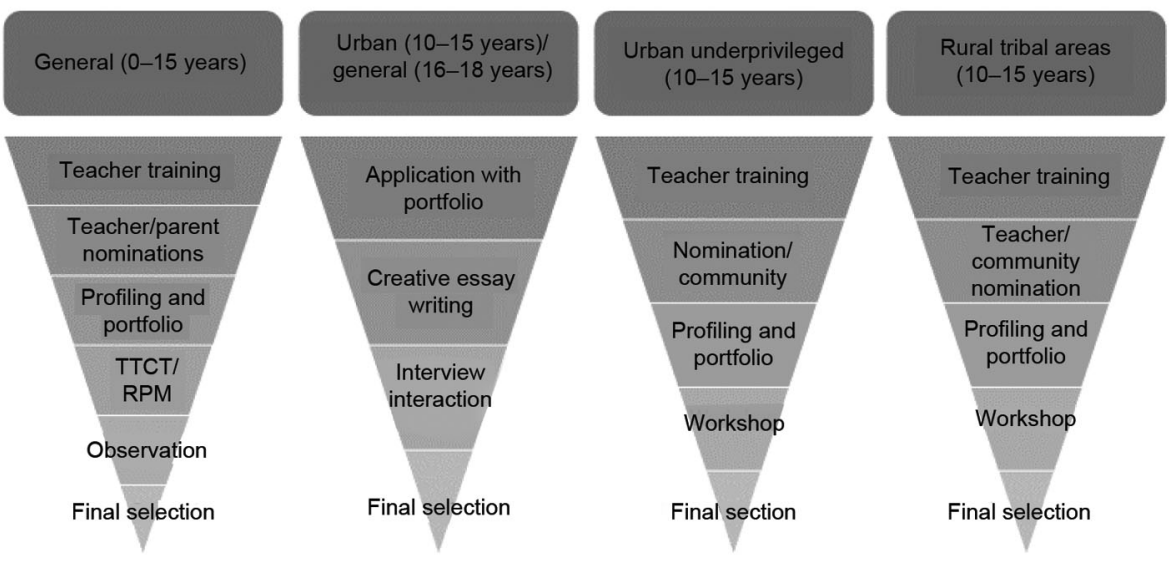

Figure 1. Identification protocols for gifted children in India.

are significant in identifying and providing support to them.

The Portfolio provides data points on specific interest areas through records of the following:

- Audio and visual recordings of the child.

- Personal dairies.

- Written documents/drawing samples/models/experiments.

- Participation/prizes in competitions/school day/sports/ cultural activities.

Based on the above data children are shortlisted and administered the Raven's Progressive Matrices9 (RPM) with Indian norms and Torrance Test for Creative Thinking $^{10}$ (TTCT; Indian norms developed by NIAS). Students who score above the 95th percentile in both these tests are identified as gifted. In a majority of cases, nominations from teachers are preceded by comprehensive training provided by NIAS. The institute has published a Teachers' Training Manual in English and Kannada to identify gifted children. The Manual is a result of a series of training workshops conducted for teachers from across the country.

\section{NIAS-supported Advance Learning Centres (10-15 years)}

In an attempt to expand the programme and focus on building a sustainable supplementary mentoring programme, NIAS-supported Advanced Learning Centres (ALCs) were launched in Bengaluru and Hyderabad. Recognizing the challenge of scale in India, the identification process was modified to include creative essay and interaction rounds. Just like in the NIAS General Pool, teachers from neighbourhood schools are trained and student nominations are solicited through the student nomination behavioural rating scale. The students nominated by the teachers have to fill a detailed application form that solicits data on their academic performance over the preceding three years and other data for their case studies and portfolios. All students nominated by the teachers have to participate in a creative essay round that provides them with the opportunity to offer innovative and unique solutions to long-standing problems in society, spanning across science, technology, social environmental and political fields. In addition, the student has to identify a subject of his/her interest and provide a comprehensive narrative of activities undertaken by him/her in pursuing this interest as well as a personal roadmap of the future of his/her area of interest.

The essay is evaluated by the NIAS team with respect to creativity, innovation, passion and commitment of the student. The shortlisted students are further screened in an interaction round by a team of experts from different fields, to corroborate their deep learning, creativity, innovation and passion.

Based on their scores in the interaction round, students are selected for the ALCs. These students participate in weekend classes of 3-5 h, where they are provided with opportunities to acquire advanced learning in a range of topics beyond the classroom and engage in project work in their areas of interest. These students are mentored by student mentors and later by experts. Summer and winter residential workshops on contemporary topics are organized for them. Monthly lecture series and an annual exhibition - ALCHEMY where selected project works are exhibited are also part of the mentoring programme. Internship opportunities are provided to the students by linking them with mentors from premier institutions. Currently, there are two ALCs in Bengaluru South and Bengaluru North, and one in Hyderabad. The partnering schools that provide infrastructure support to the programme are Silveroaks International School, Bengaluru 
and Hyderabad and Vidyashilp Academy International School, Bengaluru. There are 150 students in the programme.

\section{NIAS PRODIGY MAIYA Fellowship Programme (16-18 years)}

The NPMFP initially targeted tenth-grade students who passed the State Board exams of Karnataka. NIAS obtains the list of the top 500 boys and girls separately from rural and urban areas from the State Board. Information sought also includes the name and mobile number of the students, the name and address of their schools and mobile number of their headmaster/mistress. Efforts are made to reach out to students in rural and urban areas by posting the announcement and application forms in schools where the students have completed their tenth grade. The team follows up with the students through mobile numbers to encourage them to apply for the fellowship programme. However, the application is open to all students who have scored over $80 \%$ in their tenth grade during the year of announcement of the fellowship programme.

All applicants are invited for a creative essay round in Bengaluru. The questions in the essay round are similar to those used for the ALCs. The essays are scored to measure deep learning, creativity, innovation, ability to connect with what one has learnt and articulation, among others. Each essay is independently scored by more than two members. The shortlisted students then attend an interaction round with a team of experts from different disciplines. The students are evaluated on their deep learning, passion, creativity and innovation during the interaction round. Shortlisted students are selected for the NPMFP, which includes a onetime prize amount of Rs 50,000 and provision of mentoring for at least five years. Annual mentoring workshops with experts from India and abroad are organized for the fellows every year. The NIAS team actively works with the students to connect them with mentors based on their areas of interest. Counselling support for psychosocial issues as well career aspirations is provided through a broad-based network developed by NIAS. The programme was started in 2017 and currently has 60 students.

\section{NIAS-supported Advanced Learning Centres for the urban poor (10-15 years)}

The NIAS ALC which is in its third year of operation quickly realized that the students of the centre largely belong to the middle- and upper-middle class. To enable mid-course correction, the team partnered with Parikrma, an NGO that works with the rural poor in Bengaluru. The NIAS team trained teachers of the four Parikrma Centres of Learning and students were nominated by the teachers.
A three-day residential workshop was organized for the selected children who were provided activities that allowed them to demonstrate behaviour associated with giftedness. Using multiple observers, students were shortlisted for the ALCs.

\section{NIAS Programme for Rural and Adivasi Communities (10-15 years)}

A TCS grant and collaboration with the Education Department of the Government of Karnataka provided us with an opportunity to work in the rural and tribal areas of the Chamrajnagar and Mysuru districts in the state, during 2016-18. In collaboration with the district-level officers of the Education Department, NIAS trained over 1000 teachers from the above two districts through several workshops. The teachers trained in the programme nominated students using the teacher nomination behavioural rating scale. The teacher nomination was screened and over 175 students were shortlisted for detailed case studies and profiling. Through an intensive training of field investigators, the NIAS team collected data from 175 students through field visits and interactions with their families and communities. The data covered information required for the case profiles and portfolios described in the previous sections. The students were invited to a three-day workshop which provided them with opportunities to demonstrate their giftedness. Based on observations by multiple observers, 100 students were selected for the mentoring programme. This programme will have to recognize that distance between habitats and schools in rural and tribal areas is a real challenge.

\section{Multi-level multi-stage mentoring models}

India is a country that reflects wide-ranging variations in resources at the community and school levels. The mentoring model developed by NIAS is cognizant of these inequalities, and therefore, the NIAS-EGT has developed a multi-stage, multi-level mentoring model that reflects the diverse Indian context.

\section{Mentoring model for India}

Figure 2 highlights the mentoring model for India.

Mentoring of children between 0 and 9 years from urban and rural areas: For young children of 0-10 years, the NIAS team works with parents of the gifted children to develop individual plans for mentoring, which are implemented with a high degree of parental support. Awareness and interactive workshops are organized for the parents, with experts from the fields of psychology and education. These workshops seek to train the parents to 


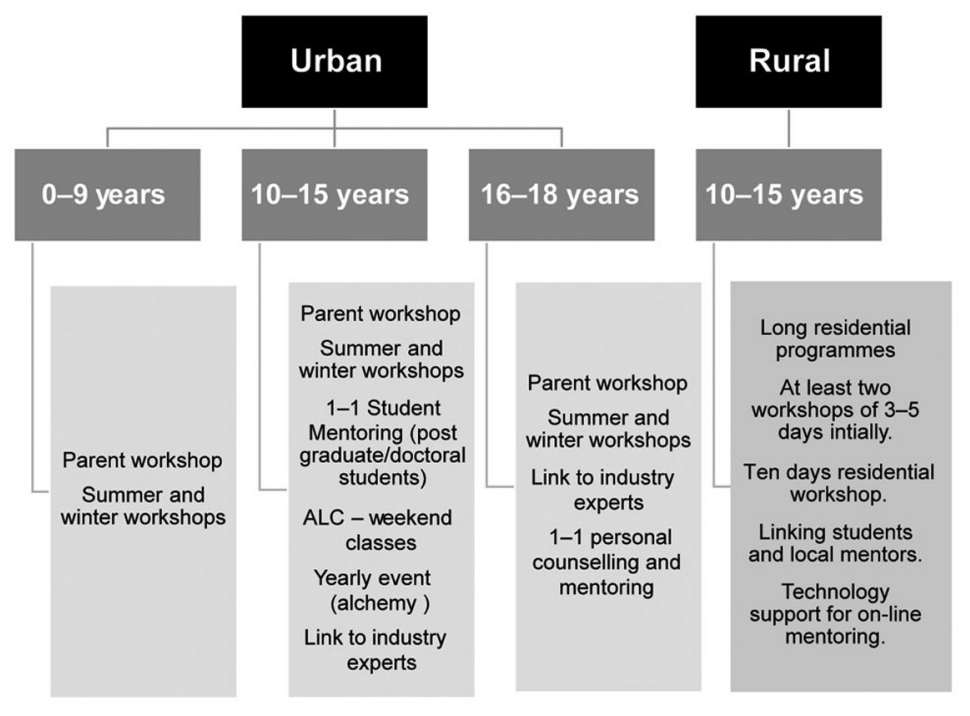

Figure 2. Mentoring mechanisms for gifted children in India.

play a facilitative role in the early years of development of their child. NIAS through its network of counsellors provides support to the parents and children during their formative years. Parents and children are engaged in a series of discussions about the choices available for schooling and curricula such as for example, home schooling. Annual summer and winter workshops of 3-5 days are organized to facilitate peer interactions for the children and to provide them with exposure to foundational skills beyond their grades in order to assist progress in their areas of interests. On-line platforms such as WhatsApp groups and group e-mails are used to share information regarding existing opportunities for children in that age group. Resources and experiences are also shared through these platforms.

Mentoring of children between 10 and 15 years from urban areas: In addition to the provisions for mentoring children in the age group of $0-10$ years, those in the $11-$ 16 years group can enrol in the ALCs. NIAS organizes weekend classes of 3-5 h at the ALCs which are located in some schools, and ideally seeks to serve gifted children from the neighbourhood schools. The Centres provide advanced learning curriculum in broad areas of interest of the students beyond their school curriculum. Monthly lecture series by experts are organized to expose the students to contemporary areas of knowledge. The students engage in project- based learning during the year. Selected projects are displayed during the annual ALCHEMY organized at NIAS during the Open Day of IISc. As the students engage in deep learning in their areas of interest, acquire the vocabulary of their disciplines and demonstrate progress, they are linked to student mentors. These student mentors are graduate or doctoral students from premier institutions with similar subject expertise. Through the process the ALC students are thus connected to student mentors who work in the same areas.

Mentoring of children between 17 and 18 years from rural and urban areas: The fellows of this programme participate in annual residential mentoring workshops, where they meet national and international experts from different fields who share their current research interests. Students present their own projects that they are currently engaged in and receive feedback from the experts. At the end of the workshop, the students are linked to one or more mentors with whom they interact as they make choices for their educational and career paths. All students are linked through a technology platform to share information, concerns and experiences. It is useful to employ technology to leverage mentoring beyond state and national boundaries.

Mentoring for children between 11 and 15 years from rural and Adivasi communities: The identification and mentoring of gifted children from rural and Adivasi communities requires active collaboration with the state and central governments. The biggest challenge that we face is the distance between the schools and communities. The fact that most of these children have never left their villages, poses a serious challenge to organizing residential workshops even at the district level. Hence it becomes necessary to collaborate with the Education Department at the district, taluk and village level to provide the necessary financial and social support to these children. Through a gradual process, one may need to move from 3 to 5 and then ten-day residential workshops to gain their confidence. Long residential workshops are the most effective form of providing mentoring support 
Box 1. Case study of a gifted child.

XXX was identified by NIAS-EGT when he was seven years old. The interaction with the child and his parents resulted in him moving to a school that practised differentiated learning through the IB curriculum. He was part of the earlier summer/winter workshops organized for children identified in the programme. Through a mentor-mentee workshop organized at NIAS with graduate and doctoral students, XXX was paired with a student mentor from the Indian Institute of Science (IISc), Bengaluru. Over a period of more than seven years interaction with the student mentor, XXX progressed in deep learning of mathematics, physics, chemistry and biology, and identified deep interest in the field of environmental science. The student mentors introduced him to scholars in his interest areas. Throughout this period, XXX interacted with three student mentors from IISc and visited their laboratories during his summer vacation. In 2017, on IISc Open Day, he met the faculty of the Department of Environmental Sciences and was offered an internship. XXX has completed two stints of summer internships when he was in grades 7 and 8 respectively. He proceeded to work on a project on clean air titled 'Designing algal microcapsules for efficient carbon sequestration through photosynthesis'. The project was displayed in the annual ALCHEMY event organized by NIASEGT in 2018. This involved more than three years of data collection, study and a great insight into research and publication. The paper XXX wrote on his project work was published in Young Scientist Journal (https://ysjournal.com/designing-algal-capsules-for-carbon-sequestration/). He is now working towards publication of his paper in Resonance. He is also currently working on the prototype of a device based on his project, that can be attached to vehicles to sequester carbon from the exhaust. A glimpse of the profiles of children in the NIAS-EGT is available at http://prodigy.net.in/student-profile-list.

for children from these communities. NIAS proposes a ten-day residential workshop in summer and winter to mentor these children. The workshop aims at providing broad guidelines to pursue deep learning and engagement in project-based work. Teachers from schools can play a facilitative role in connecting the students to mentors in their local areas. There is a need to develop a network of available mentors in local communities. Mapping locallevel mentors with gifted children during the initial phase is critical to provide direct mentor-mentee interaction. Technology support will be vital to linking gifted children with experts at the state and national levels. The exposure visits of gifted children from rural and tribal areas is essential to broaden their horizons and enable them to experience different learning environments. Such tacit learning is necessary for their enrichment, and new environments which provide great opportunities need to be integral to the mentoring process. Technology can be leveraged to access experts at state, national and international levels. Students must be provided with technology devices and training in their use, to connect them to human resources and learning material. The provision of translation services is integral to the effectiveness of the mentoring process. Counselling support is critical to sustain the motivation and commitment of the gifted children from these communities, given the multiple challenges they face in their day-to-day lives (Box 1).

\section{Conclusion}

Research worldwide on the identification of gifted and talented children has predominantly used psychometric measurements. In an effort to increase representation of the gifted children from diverse backgrounds, researchers in the field have attempted at broader conceptualization of giftedness ${ }^{11,12}$. Though representation has increased using non-verbal assessments, it has not been entirely successful. Multiple tools encompassing observation of student behaviour in learning situations, performancebased and portfolio approaches amongst students with relatively similar resources at school and home are gaining popularity ${ }^{13}$. For gifted children from disadvantaged communities, there is a need to recognize the interplay of multiple factors that are integral to identification and mentoring. These include intelligence, creativity, motivation, passion and resilience, social and cultural factors, and more importantly, the environment that provides them the opportunity for recognition and mentoring, among others. All these factors interact in differential ways. It is discerning these patterns through the analysis of a large database that may be necessary to address the under-representation of children from disadvantaged communities in programmes of education for the gifted and talented. For countries like India that have vast segments of populations with impoverished home environments, early identification of gifted children is an added advantage ${ }^{14}$. India can be a leader in setting this new direction for developing countries with limited resources, both financial and human.

1. NAGC, Key considerations in identifying and supporting gifted and talented learners - a report from the 2018 NAGC Definition Task Force. National Association for the Gifted Children, Washington DC, 2019; https://www.nagc.org/sites/default/files/ Position\%20Statement/Definition\%20of\%20Giftedness $\% 20 \%$ 282019\%29.pdf (accessed in September 2020).

2. Davalos, R. and Griffin, G., The impact of teachers' individualized practices on gifted students in rural, heterogeneous classrooms. 
Roeper Rev., 1999, 21(4), 308-314; doi:10.1080/02783199909553982.

3. Neihart, M. et al. (eds), The Social and Emotional Development of Gifted Children: What do we know? Prufrock Press, Waco, TX, USA, 2002.

4. MHRD, National Education Policy 2020, Ministry for Human Resource Development, GoI, 2020, p. 65; https://www.mhrd. gov.in/sites/upload files/mhrd/files/nep/NEP Final English.pdf (accessed in September 2020).

5. NIAS, Teacher nomination behavioural rating scale. National Institute of Advanced Studies, Bengaluru, 2013; http://prodigy. net.in/nomination_form (accessed in September 2020).

6. Smutny, J. F., Teaching young gifted children in the regular classroom, 2000; https://files.eric.ed.gov/fulltext/ED445422.pdf (accessed in September 2020).

7. Gross, M. U. M., Small poppies: highly gifted children in the early years. Roeper Rev., 1999, 21(3), 207-214; doi:10.1080/ 02783199909553963.

8. Pfeiffer, S. I. and Petscher, Y., Identifying young gifted children using the gifted rating scales - preschool and kindergarten form. Gifted Child Q., 2008, 52, 19-29; doi:10.1177/0016986207311055 [Crossref], [Web of Science ${ }^{\circledR}$ ], [Google Scholar].

9. Raven, J. C., Court, J. H. and Raven, J., Manual for Raven's Progressive Matrices, Oxford Psychologists Press, Oxford, UK, 1998.

10. Paul, T. E., Torrance Tests of Creative Thinking, Personnel Press, Princeton, NJ, USA, 1966.
11. Ford, D. Y., Multicultural gifted education: a wakeup call to the profession. Roeper Rev., 1996, 19, 72-78.

12. VanTassel-Baska, J., Patton, J. M. and Prillaman, D., Gifted youth at risk: a report of a national study. The Council for Exceptional Children, Arlington, VA, USA, 1991.

13. Karnes, F. A., State definitions for the gifted and talented revisited. Except. Child., 2000, 66, 219-238.

14. Kurup, A. and Maithreyi, R., A review of challenges in developing a national program for gifted children in India's diverse context. Roeper Rev., 2012, 34(4), 215-223.

ACKNOWLEDGEMENTS. The NIAS-EGT was initiated in 2011 by Prof. R. Chidambaram (Principal Scientific Advisor, Government of India). I acknowledge the contributions of Programme Advisory Committee members, Directors of NIAS - Prof. Shailesh Nayak, Prof. V. S. Ramamurthy and late Prof. Baldev Raj; Ajay Chandra, Parvathy Jayan, Jena, Dr Shalini Dixit, K. Ashwini, Surbi Arora, C. Pritha and H. Sudarshan-members of the team during this 2011-2020. The Project was financially supported by the Office of the Principal Scientific Advisor, Government of India (2011-15) and Tata Consultancy Services (2016-19).

Received 4 September 2020; revised accepted 5 October 2020

doi: $10.18520 / \mathrm{cs} / \mathrm{v} 120 / \mathrm{i} 3 / 472-478$ 